\title{
Experimental Study of the Transient Response of Bunsen Flame to Nanosecond Pulsed Discharges
}

\author{
Shaohua Zhang ${ }^{1} \cdot$ Xilong Yu $^{1} \cdot$ Heng Xiong $^{1} \cdot$ \\ Hui Zeng ${ }^{1} \cdot$ Fei Li $^{1}$
}

Received: 29 September 2014/ Accepted: 22 July 2015/Published online: 9 August 2015

(C) Springer Science+Business Media New York 2015

\begin{abstract}
The transient processes associated with the interaction of a Bunsen flame and nanosecond pulsed discharges (NPD) are explored experimentally with two optical methods. A nanosecond-gated schlieren system is employed to image the shockwave propagation and the hydrodynamic response of the flame to NPD while the time-resolved optical emission spectroscopy measurements are carried out to determine active species and temperature in the plasma region created by the discharges. Therefore, the unsteady process of the interaction of the flame with the discharges is recorded in real-time by the combined measurements. Numbers of experimental evidences for understanding the dynamics of non-equilibrium plasma produced by NPD and performing further numerical simulation are offered.
\end{abstract}

Keywords Nanosecond pulsed discharges (NPD) - Time-resolved optical emission spectroscopy $\cdot$ Schlieren

\section{Introduction}

Plasma-assisted combustion is a promising way to enhance flame velocity and reduce pollutant emissions [1-3]. Because of the vital practical significance to alleviate the resource and environmental problems, a variety of plasma generator systems including dielectric barrier discharges (DBD) [4], corona discharges [5], and microwave discharges [6], and the nanosecond pulsed discharges (NPD) [7] have been used to explore plasmaassisted combustion. In these systems, NPD have received special attention due to high energy efficiency and low power consumption. Similar to laser induced breakdown

Xilong Yu

xlyu@imech.ac.cn

Shaohua Zhang

shzh@imech.ac.cn

1 State Key Laboratory of High Temperature Gas Dynamics, Institute of Mechanics, Chinese Academy of Sciences, Beijing 100190, China 
spectroscopy $[8,9]$, nanosecond pulsed discharges can produce large amounts of neutral molecules, atoms, radicals, and other active species in mixtures within combustion in nanosecond scale which is shorter than chemical reaction time mostly. Therefore, wellknown crucial activated particles can initiate chain reactions, shift chemical equilibrium and accelerate chemical reactions greatly, even at low concentrations. Consequently, the NPD technique has been applied to decrease the lean flammability limit $[10,11]$, increase the laminar flame speed $[12,13]$, or reduce the ignition delay time $[14,15]$ in the past decades. Except for studies that aimed to determine the ignition delay, most of the previous researchers investigated the influence of various parameters, such as pressure, temperature, fuel, burner geometry, electrode geometry or other plasma properties from chemical and thermal activation in stationary conditions. The dynamic and hydrodynamic responses of the flame to nanosecond pulsed discharges, which are very important to clarify theoretical mechanisms or to perform numerical simulation, are largely unknown.

The two-step ultrafast mechanism proposed by Popov [16] initially is the most popular approach for interpreting kinetics of electronically excited states in non-equilibrium plasmas induced by NPD. The mechanism is composed of the following two steps,

$$
\begin{aligned}
N_{2}(X)+e & \rightarrow N_{2}(A, B, C, \ldots)+e \\
N_{2}(A, B, C, \ldots)+O_{2} & \rightarrow N_{2}(X)+O+O+\text { heat } \cdot \text { release }
\end{aligned}
$$

According to the ultrafast mechanism, nitrogen molecules are firstly excited by electron impact to electronic states such as $A^{3} \Sigma, B^{3} \Pi$, and $C^{3} \Pi$ during voltage pulses. Then, the excited $\mathrm{N}_{2}$ molecules are quenched by oxygen molecules, producing atomic oxygen and heat release in the afterglow. In previous published studies, the two-step process mechanism was partial verified by the experimental observation of the density temporal evolution of $\mathrm{O}$ atom at the ground state [17] and the measured high rotational temperature of $\mathrm{N}_{2}$ with the assumption that rotational and translational modes of $\mathrm{N}_{2}$ are equilibrated at all times [18]. However, the overview of recent experimental and kinetic modeling studies of lowtemperature plasma-assisted combustion by Adamovich et al. [19] exhibits the key challenges like the development of a predictive plasma-assisted combustion chemistry mechanism requires a set of temperature and radical concentration data during NPD process. The results would help quantify the effect of 'rapid' heating and the possible reactions of vibrationally excited molecules. Furthermore, more plenty of transient

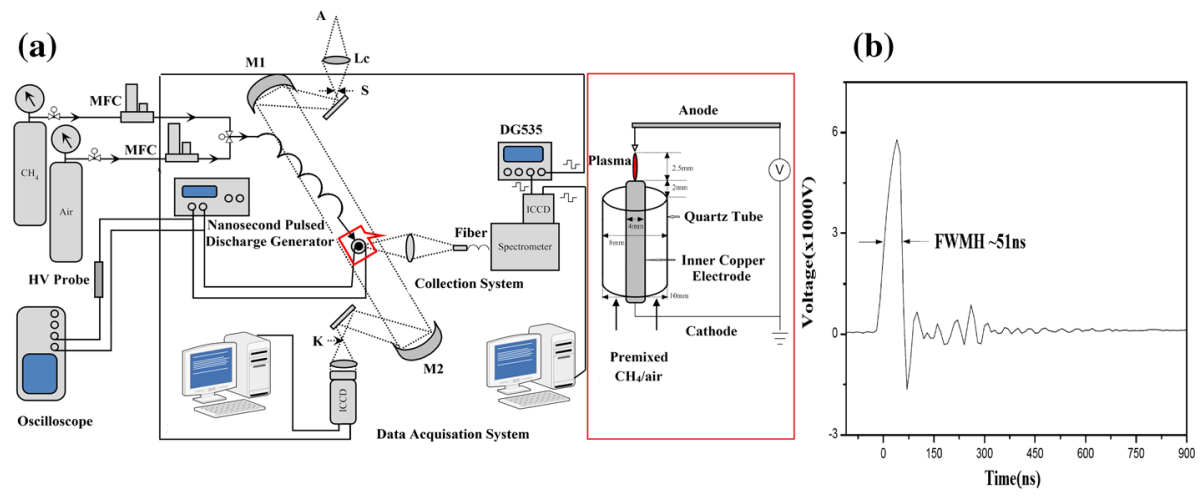

(b)

Fig. 1 a Schematic diagram of experimental setup, $\mathbf{b}$ a typical waveform of NPD 
information about the process are needed to prove or supply the mechanism and to understand the non-equilibrium process of NPD.

Therefore, in the present work, we present a fundamental study investigating the transient interaction of a premixed $\mathrm{CH}_{4}$ /air Bunsen flame with NPD by a nanosecond-gated schlieren system and time-resolved optical emission spectroscopy. The former optical technique, schlieren technique, has been used widely to observe inhomogeneities of transparent media like air or water, based on the refraction of light by the refractive index gradient. Here, it was employed to image the shockwave propagation and the interaction process of the flame and the discharges while the latter one was carried out to monitor active species and temperature evolution in the process. To our knowledge, both of the two techniques have rarely been used to understand the nanosecond pulsed discharges.

\section{Experimental Setup}

Figure 1a demonstrates a schematic diagram of the experimental setup. In a typical experiment, the high-voltage (HV) signals used for discharging were supplied by a homemade HV alternating current transformer (based on the ET series $2000 \mathrm{~W}$ Regulated High Voltage DC Power Supplies, Glassman High Voltage Inc.) and monitored by an HV probe (Tektronix P6015A) combined with a digitizing oscilloscope (Tektronix DPO4032). The premixed gas sample containing methane and air with a suitable equivalence ratio, supplied from cylinders through stainless steel pipes and controlled by individually calibrated mass flow controllers (SLA5851S Thermal Mass Flow meters, BROOKS Instrument), flows through distant pipelines and the annular space between the inner electrode and the quartz tube of the Bunsen burner. The mass flow meters controlled the gas flow precisely, while the distant pipelines ensured that gases were mixed sufficiently. The nanosecond pulsed discharges were applied on the premixed Bunsen burner schematically presented in the insert of Fig. 1a. The Bunsen burner was a coaxial configuration composed of an inner copper stick whose diameter is $4 \mathrm{~mm}$ and an outer quartz tube with an inside diameter of $8 \mathrm{~mm}$ and the thickness of $1 \mathrm{~mm}$. The copper stick was employed as the cathode electrode (grounded) of the NPD. The tungsten anode was a cylindrical electrode which has a diameter of $1 \mathrm{~mm}$ with a sharpened tip. In discharges, the inter-electrode gap was set at $2.5 \mathrm{~mm}$. At the same time, a positive high voltage (HV) pulse of $5.8 \mathrm{kV}$ was applied to the electrode tip while the Full-Width-Half-Maximum (FWHM) duration of the $\mathrm{HV}$ pulse is about $51 \mathrm{~ns}$ generally as shown in Fig. $1 \mathrm{~b}$.

For the measurement of the schlieren photography, a pulsed xenon flash lamp with duration of $800 \mu$ s was used. The schlieren set-up consisted of achromatic type lenses with a diameter of $150 \mathrm{~mm}$ and a focal length of $1500 \mathrm{~mm}$. The burner was located in the center of the two concave mirrors. Light came from xenon flash lamp and illuminated the laminar premixed $\mathrm{CH}_{4} /$ air flames. The schlieren images were recorded by an ICCD (Model: PIMax3, minimum optical gate width: $2 \mathrm{~ns}$, Princeton Instruments) with a camera lens (Casio, $f=35-135 \mathrm{~mm}$ ). The output of the ICCD was input to a digital board and then analyzed by a computer data acquisition system. The flame emission was collected by a lens system firstly, and then, transmitted into an Acton SpectraPro 2300i spectrometer (focal length $300 \mathrm{~mm}$ ) equipped with an ICCD (PI-Max, minimum optical gate width: $2 \mathrm{~ns}$, Princeton Instruments). The spectrometer was equipped with a 300 grooves $/ \mathrm{mm}$ grating and a $50 \mu \mathrm{m}$-wide entrance slit to get the emission spectra. The grating efficiency of the spectrometer (with ICCD) was calibrated using an NIST standard tungsten halogen 
lamp (Model: 63976, Oriel Instruments) and the wavelength calibration of the system was performed using a standard $\mathrm{Hg}-\mathrm{Ar}$ lamp. Experimental synchronization was controlled through a multi-channel digital delay pulse generator (Model: DG535, Stanford Research System).

\section{Results and Discussion}

\section{Hydrodynamic Processes and Structure Evolution in the Plasma Region with Schlieren Photography}

\section{Single-Shot Successive Schlieren Images of NPD in Air}

Schlieren photography technique is a simple and convenient way to measure the laminar flame speed indirectly, utilizing the deflection of parallel light rays in the presence of variable density gradients normal to the propagation of the ray.

As referred in chapter 2.3 of Ref. [20], the illuminance level in a schlieren image responds to the first spatial derivative of the refractive index (e.g. $\partial n / \partial x$ ), which may result from temperature changes, high-speed flows, or the mixing of dissimilar materials. Consequently, shock waves or heated gas front with sharp density gradients can be visualized. A shock wave is defined as an area of very high pressure moving through the media, like air, earth, or water. It is usually caused by an explosion or by an object travelling faster than sound. Although the sensitivity of shadowgraph whose illuminance level responds to the second spatial derivative (e.g. $\partial^{2} n / \partial x^{2}$ ) could be much enlarged in gas flows involving shock waves or turbulence, for weak disturbances, schlieren is more sensitive than shadowgraph because the $\partial n / \partial x$ usually larger than $\partial^{2} n / \partial x^{2}$ in the situation.
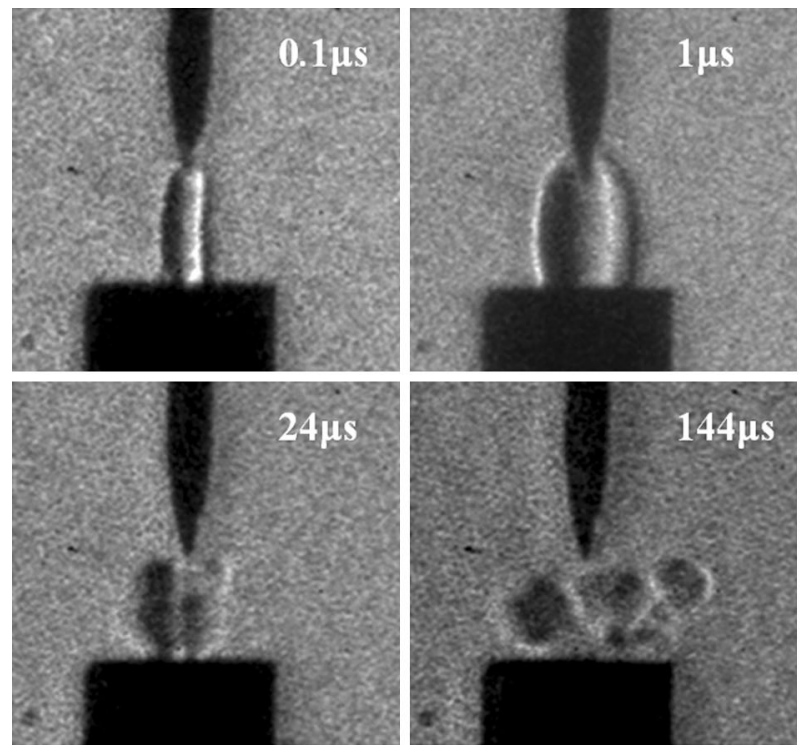

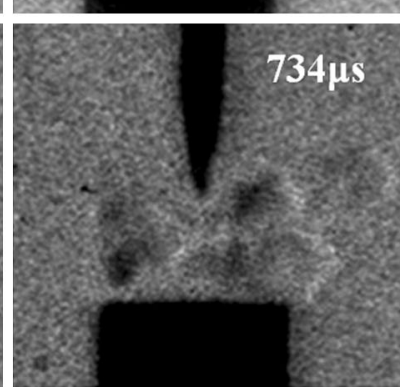

Fig. 2 Typical schlieren images of single-shot nanosecond pulsed discharges in air. The exposure time is 200 ns. Numbers upper the images represent the acquisition time after discharges initiation (delay time) 
Thus, in the present experiment, we employed the nanosecond-gated schlieren system to monitor the flow disturbances of the flame with NPD. The successive schlieren images of shockwave propagation induced by the single-shot nanosecond pulsed discharges in air were recorded and displayed in Fig. 2.

It noted that initially the shock-wave accompanies the heated gas channel growth in a cylindrical shape similar to Ref. [20]. It means that, the NPD leads to a rapid increase in pressure and temperature in the gas channel forming high-temperature high-pressure plasma channels at first during the discharges. While the discharges energy is transferred into the excitation of internal degrees of freedom in the gas (like the electronic excitation of $\mathrm{N}_{2}$ molecules et al.), a portion of the discharges energy is rapidly converted to the energy of translational degrees of freedom. The fraction of transmitted energy varies from 10 to $60 \%$ at different electric field [21]. The process of energy transfer and relaxation occurs in a very short space of time, like the rotational-translational (RT) relaxation process requires only a few collisions. The typical relaxation time is comparable to the gaskinetics time, within a nanosecond under normal conditions. The vibrational-translational (VT) relaxation time under low-temperature conditions is usually in microseconds when the mixture contains significant amounts hydrocarbons [2]. The overheated high-pressure plasma channels begin to expand into the surrounding atmosphere forming a strong cylindrical shock wave by compressing the ambient gas at its front into a thin shell just like in Fig. 2 (delay $=0.1 \mu \mathrm{s}$ ). Next, the hot, low-density gas of the plasma channel pushes the cold, high-density gas out, causing the propagation of the shock wave. When the shock wave propagates beyond the heated gas channel, it changed from a cylindrical shape to a spherical wave as shown in Fig. 2 (delay $=6 \mu \mathrm{s}$ ). Finally, the spherical-shape shock wave will degenerate into a sound wave, according to the calculated shock wave velocity that will be displayed below. Afterwards, after a long time (hundreds of microseconds), the vortexes and turbulization capable of stirring gases in the discharges area appear. It should
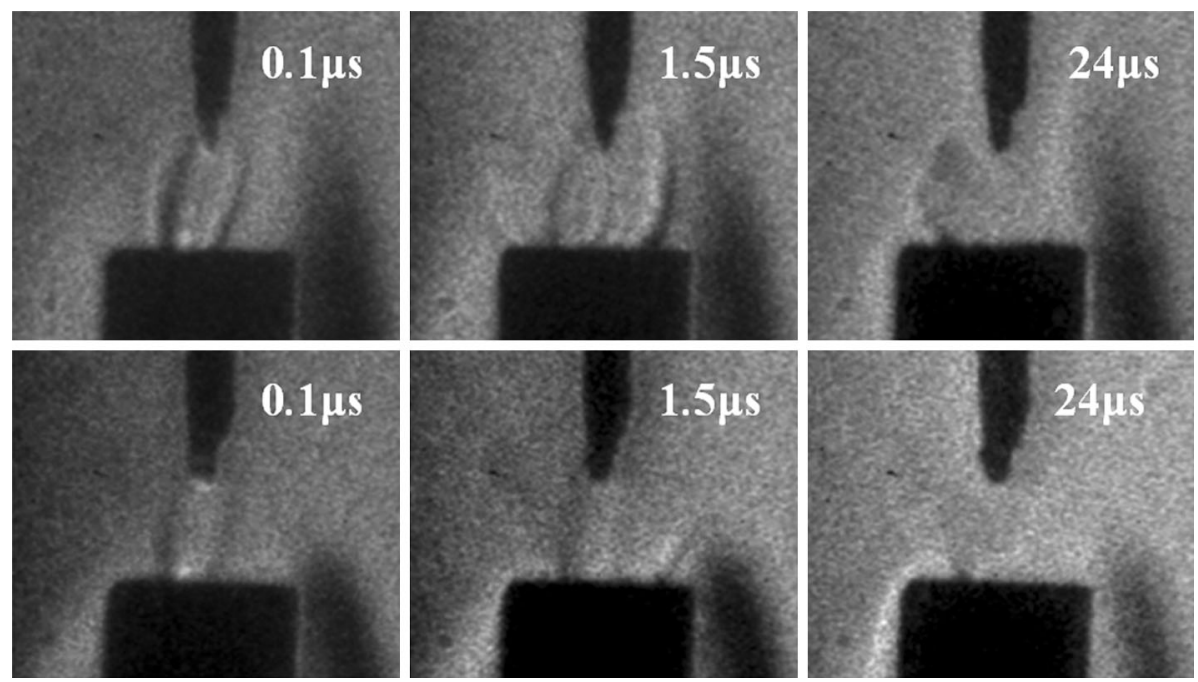

Fig. 3 Typical schlieren images of nanosecond pulsed discharges in the premixed $\mathrm{CH}_{4} /$ air Bunsen flame (upper row $\Phi=1.27$, lower row $\Phi=0.85$ ). The exposure time is $200 \mathrm{~ns}$. Numbers upper the images represent exposure time after discharges initiation 
be induced by the initial overexpansion of the hot gas channel and the reverse flow caused by the electrodes of the discharges.

\section{Schlieren Images of NPD in Bunsen flame}

For the purpose of exploring the interaction of combustion with the NPD, the single-shot nanosecond pulsed discharges were applied to two premixed methane/air Bunsen flames with different equivalence ratio (1.27, 0.85 respectively). Figure 3 showed that successive schlieren images of the shockwave propagation and the interaction process of the singleshot nanosecond pulsed discharges with the flame. Similar to the discharges study in preheated air flow [22], the distinct microstructure of the discharges area was smoothed greatly in the hot gas. The cause of the reduction might be low threshold value of breakdown of NPD in the hot gas. Less energy of discharges would be absorbed by the preheated molecules or active species in combustion than unheated molecules, due to the thermal population in the flame. Therefore, the sharp density gradients of shock waves in Fig. 2 were weakened greatly in the flame as shown in Fig. 3. Moreover, the schlieren images also demonstrated that the reduction in small flame who owns lower equivalence ratio is more obvious. We think that this phenomenon is resulting from the small recirculation area which is deemed to the unburned inner flame in a Bunsen flame. Comparing with the radius of the shock wave in flame with that in air at the same delay time, it can be found that the propagation of the shock wave was greatly speeded up. The acceleration will be confirmed and quantized by the measurement of the shock wave speed as shown in the next section.

\section{The Measurement of the Shock Wave Velocity}

With the schlieren images of NPD in Figs. 2 and 3, the temporal evolutions of the shockwave radius in the middle plane of the gap were measured. Then, the shock-wave velocity was deduced from the measured radius and the delay time. In Fig. 4, the measured shockwave velocities in the three mentioned cases were exhibited. It could be found that the shock wave velocities in three cases are all decreased rapidly and exponentially with the delay time and the shock-wave radius.

Fig. 4 The measured radial velocity of shock wave produced by NPD in air and in flame

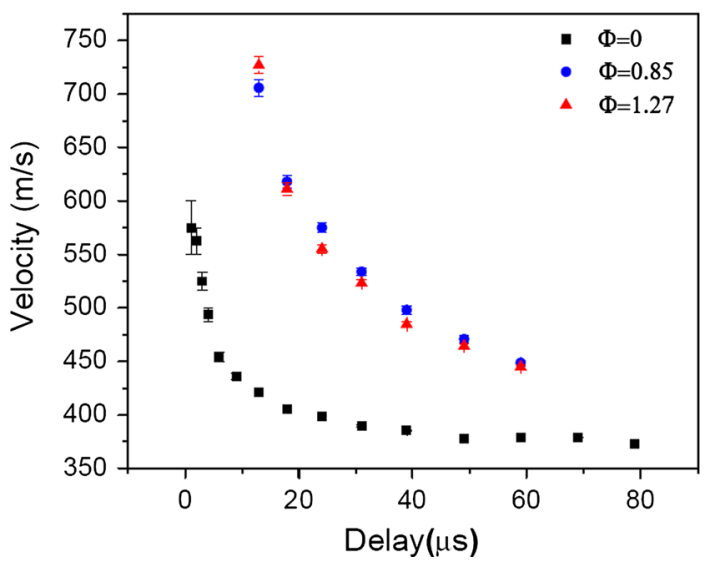


In air $(\Phi=0)$, the radial velocity of the shock wave ranges from about 575 to around $372 \mathrm{~m} / \mathrm{s}$ during the discharges and its afterglow. Depending on the relation of temperature and sound speed, the value of the speed of sound in air at $300 \mathrm{~K}$ is $347 \mathrm{~m} / \mathrm{s}$ that is very close to the measured shock wave velocity after the relaxation process $(372 \mathrm{~m} / \mathrm{s})$. It means that, as it propagates, the shock-wave weakens into a sound wave finally.

The sound speeds in unburned premixed $\mathrm{CH}_{4}$ /air gases and in the premixed $\mathrm{CH}_{4} /$ air Bunsen flames were also calculated by the Gaseq software in equilibrium at $300 \mathrm{~K}$ and $1 \mathrm{~atm}$. The result shows that sound speeds are 355.5 and $945.6 \mathrm{~m} / \mathrm{s}$ respectively when equivalence ratio of the premixed gases is about 1.27 while the values are 353 and $915.6 \mathrm{~m} / \mathrm{s}$ respectively in the other premixed gases $(\Phi=0.85)$. Unfortunately, in the present experiment, the shock wave was only observed in midway through the full propagation process, as well as the measured velocity because of the reduction of combustion. As shown in Fig. 4, the velocity of the shock wave decreased exponentially duo to the nonuniform high temperature within the flame and the room temperature in the outside atmosphere. However, it is obvious that the shock wave velocity was significantly speeded up by the combustion as shown in Fig. 4. Comparing with the discharges in air, the amplification of the shock wave velocity in the flame is quite obvious, even up to $75 \%$ at $16 \mu \mathrm{s}$ after discharges initiation. At that time, the measured velocities of the shock waves are above $700 \mathrm{~m} / \mathrm{s}$ in the flames with different equivalence ratio. The values are consistent to that in the investigation of laser induced plasmas on non-energetic and energetic materials by Gottfried [23] in which the excitation of energetic materials generates faster external shock front velocities $\left(>750 \mathrm{~m} / \mathrm{s}^{-1}\right)$ compared to non-energetic materials $\left(550-600 \mathrm{~m} / \mathrm{s}^{-1}\right)$ while the shock front velocities of non-explosive materials is about $650-700 \mathrm{~m} / \mathrm{s}^{-1}$. In the investigation, it had been proved that the thermal energy in energetic materials was caused by both the energy transferred from the laser and the subsequent exothermic chemical reactions of the ablated species. And, the addition energy release to the plasma through the exothermic chemical reactions of energetic materials produced significant increases in shock velocity. Thus, in the present experiment, the enhancement of the shock wave velocity in the Bunsen flames of $\mathrm{CH}_{4} /$ air, can be contributed to the exothermic chemical reactions initiated by the nanosecond pulsed discharges with considering the $\mathrm{CH}_{4}$ gas as the energetic materials. From a fundamental kinetics perspective, the enhancement of the shock wave velocity in combustion might contribute to the faster relaxation process of the excited particles in flame than in air. For instance, according to the data tabled in Ref. [2], the rate coefficients of vibrational-translational relaxation of vibrational excited nitrogen molecules $\mathrm{N}_{2}(v)$ with $\mathrm{N}_{2}$ and $\mathrm{O}_{2}$ are rather small $\left(10^{-17} \mathrm{~cm}^{3} \mathrm{~s}^{-1}\right)$. It means the collisional relaxation time of nitrogen with oxygen is in the millisecond range even at atmospheric pressures. But the collisional relaxation time could be greatly shortened to microseconds when the mixture contains significant amounts of $\mathrm{H}_{2}$ or hydrocarbons duo to the rather high rate coefficients of VT relaxation of $\mathrm{N}_{2}(v)$ with $\mathrm{H}$, $\mathrm{O}$, OH et al. (three or fourth orders of magnitude than that of $\mathrm{N}_{2}$ or $\mathrm{O}_{2}$ ).

As a conclusion, the microstructure and hydrodynamic responses of the flame to nanosecond pulsed discharges were explored by schlieren images. The experimental results demonstrated that the NPD leads to a rapid increase in pressure and temperature in the gas channel at first, as predicted by the two-step ultrafast mechanism. The overheated highpressure gas channels began to expand into the surrounding space forming a strong cylindrical shock wave. Then the shock wave propagated beyond the heated gas channel to constitute a spherical wave. At last, it degenerated into a sound wave. The measurement of shock wave velocity showed that the velocities decrease exponentially with the delay time and the shock-wave radius. And, the Bunsen flame accelerated the propagation of the 
shock wave greatly. These experimental results displayed that NPD could generate shock waves and vortices by heating and compressing the gas in the discharges area within a rather short time (short than $100 \mathrm{~ns}$ in this study). The ultrafast heating, the generated shock wave and vortices may have important applications in aerodynamic flow control, plasma-assisted combustion, or gas treatment.

\section{The Determination of the Active Species, Their Evolutions and Temperature by Time Resolved Optical Emission Spectroscopy}

\section{The Emission Spectra of Bunsen Flame, NPD in Air, and NPD in Flame}

In order to determine the intrinsic flame characteristics with NPD and to study the kinetics of the produced active particles capable of affecting combustion, the emission spectra of three different cases in the range of $300-800 \mathrm{~nm}$ : case 1, the flame optical emission spectroscopy, case 2, the spectrum of nanosecond pulsed discharges in air, case 3 , the spectrum of the flame with NPD, were recorded with millisecond exposure time $(500,2$, $1 \mathrm{~ms}$ respectively) and displayed in Fig. 5.

With analyzing the spectra in Fig. 5, it can be found that the emissions of the premixed $\mathrm{CH}_{4}$ /air Bunsen flame are rather weak in case 1 . Almost all emissions come from the intermediate radicals, e.g. $\mathrm{OH}, \mathrm{CH}$, and $\mathrm{C}_{2}$. In the flame emission spectrum with $500 \mathrm{~ms}$ exposure time, the $\mathrm{OH} \mathrm{A}{ }^{2} \Sigma^{+}-\mathrm{X}^{2} \Pi \Delta \mathrm{v}=0$ transition ( $\mathrm{v}$ is vibrational quantum number) around $308 \mathrm{~nm}$, the $\mathrm{CH} \mathrm{A}{ }^{2} \Delta-\mathrm{X}^{2} \Pi(\sim 431 \mathrm{~nm})$ and $\mathrm{B}^{2} \Sigma^{-}-\mathrm{X}^{2} \Pi(\sim 387 \mathrm{~nm})$, and some rovibrational bands of the $\mathrm{C}_{2} \mathrm{~d}^{3} \Sigma_{g}-\mathrm{a}^{3} \Pi_{u}$ electronic transition (the $\Delta \mathrm{v}=0$ band head is located at about $516.5 \mathrm{~nm}$ ) were observed and labeled. It should be noted that all of these weak emissions of the flame were not been observed with the discharges in a few milliseconds exposure time.

In contrast, the NPD produce large amounts of active atoms and ions whose emissions were quite strong. In case 2, according to the NIST Atomic Spectra Database, we had recognized many spectral lines of several activated atoms and ions, such as the $\mathrm{O}$ I triple lines at around $777 \mathrm{~nm}$, the lines of $\mathrm{N}$ atom $(\sim 742.3,744.2,746.8 \mathrm{~nm})$, the overlapped lines around 500 and $569 \mathrm{~nm}$ of NII (N ion) in the spectrum of NPD in air with $2 \mathrm{~ms}$ exposure time. Besides, three weak bands of the $\mathrm{C}^{3} \Pi_{u}-\mathrm{B}^{3} \Pi_{g}$ electronic transition of $\mathrm{N}_{2}$ molecules (located at about 380.5, 357.6, $337.1 \mathrm{~nm}$, respectively) were also determined. In

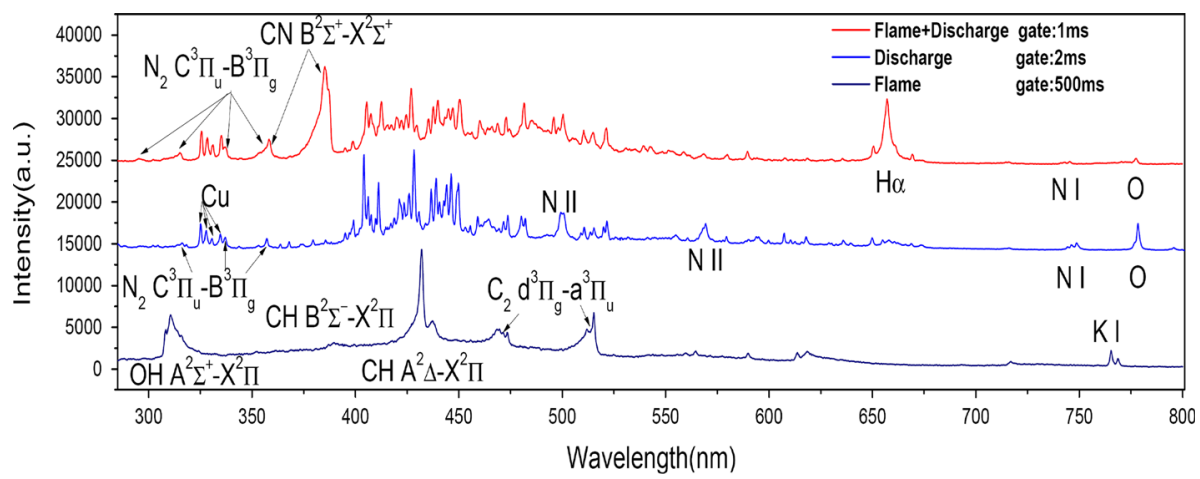

Fig. 5 Optical emission spectra in three cases, from bottom to up: 1 premixed $\mathrm{CH}_{4} /$ air Bunsen flame, 2 NPD in air, 3 NPD in flame $(\Phi=1.27)$ 
addition, we also find numerous lines of activated $\mathrm{Cu}$ atoms located at 324.7, 327.4, 330.8 and $333.8 \mathrm{~nm}$ respectively. It is believed that the active $\mathrm{Cu}$ atoms were originated from the copper cathode electrode during the discharges.

In case 3, the discharges were carried out within the Bunsen flame. Comparing to case 2, three major changes happened in the spectrum of case 3. Firstly, two new species appeared. One was the $\mathrm{H}$ atoms which came from the fuel $\left(\mathrm{CH}_{4}\right)$ in combustion, and the other one was the $\mathrm{CN}$ radicals which might be produced by dissociation and recombination process during the interaction of combustion and the discharges. Secondly, emissions of $\mathrm{N}_{2}$ molecules were enhanced greatly. Thirdly, the emissions of activated $\mathrm{N}$ and $\mathrm{O}$ atoms were reduced while the spectral lines of $\mathrm{N}$ ions vanished. It shows that the ions and atoms were involved in the chemical reactions within the flame during the discharges.

In addition, the appearance of $\mathrm{CN}$ emissions indicates the formation of gaseous $\mathrm{CN}$ radicals. It seems reasonable to assume that $\mathrm{CN}$ formation involves reactions between the $\mathrm{CH}_{4}$ fuel and the background active $\mathrm{N}_{2}$ molecules or their fragments (e.g. $\mathrm{C}_{2}, \mathrm{~N}$ atoms etc.) produced by NPD in our case. The determined interpretations require more detailed knowledge of the nitrogen atom and ion, and $\mathrm{C}_{2}$ radical, number density distributions, their respective kinetic-energy distributions, and the related reaction cross sections than can be provided simply by OES measurements.

\section{The Time Resolved Emission Spectra of NPD in the Bunsen Flame}

As mentioned above, these results of emission spectra not only displayed that the interaction of the flame and the discharges, but also offered numbers of experimental evidences for modeling theoretical mechanisms and performing numerical simulation, such as the intermediate species: activated $\mathrm{N}_{2}$ molecules, $\mathrm{O}, \mathrm{N}, \mathrm{H}$ atoms, $\mathrm{CN}$ radicals and the $\mathrm{N}$ ions. However, for modeling or verifying the dynamic mechanism of the nanosecond pulsed discharges, the time scales of the generation, evolvement and disappearance of these species might be more useful. Therefore, the time-resolved emission spectra with nanosecond exposure time were recorded, analyzed, and exhibited in Fig. 6, for determining the active species and their evolutions in the inter-electrode region produced by nanosecond pulsed discharges.

These time-resolved emission spectra of the flame with NPD were obtained with the 300 grooves $/ \mathrm{mm}$ grating and a $50 \mu \mathrm{m}$-wide entrance slit in the wavelength range of 290-400 nm. The equivalence ratio of premixed $\mathrm{CH}_{4}$ /air gas was set at 1.27 . The gate width of ICCD was programmed as $100 \mathrm{~ns}$ while the time of data acquisition varied from
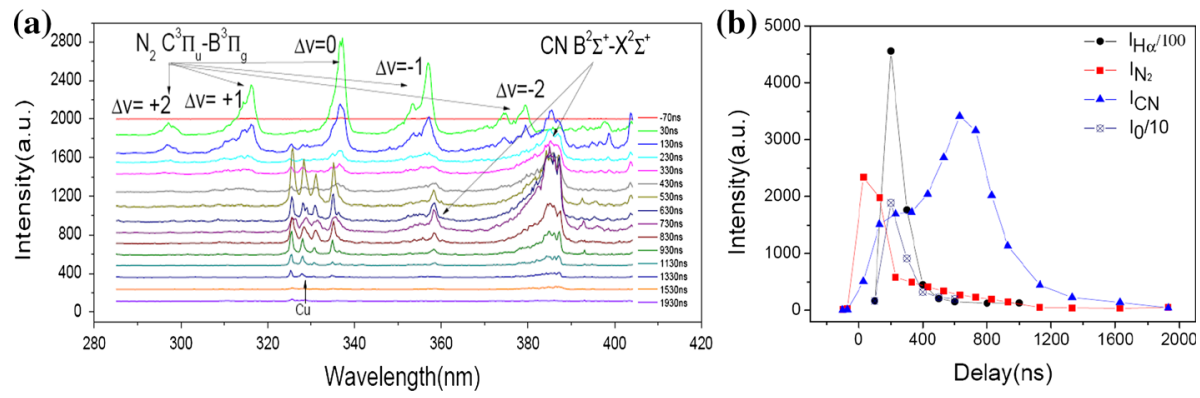

Fig. 6 a Time-resolved emission spectra of premixed $\mathrm{CH}_{4} /$ air Bunsen flame with NPD, b relaxation processes of the spectral lines of $\mathrm{H}$ and $\mathrm{O}$ atoms 
$70 \mathrm{~ns}$ before the discharges initiation $(\mathrm{t}=0)$ to $1930 \mathrm{~ns}$ after it (delay $=-70$ to $1930 \mathrm{~ns}$ ). In the time-resolved emission spectra shown in Fig. 6, we had identified the $\mathrm{CN} \mathrm{B}^{2} \Sigma^{+}-$ $X^{2} \Sigma^{+}$(the transitions are also called as the $\mathrm{CN}$ violet band) $\Delta \mathrm{v}=0$ transition (its head is located at about $388.6 \mathrm{~nm})$ and $\Delta \mathrm{v}=-1$ transition $(\sim 359.2 \mathrm{~nm})$, and a series of rovibrational spectroscopy, like some bands in $\Delta \mathrm{v}=-2,-1,0,+1,+2$ transitions of the $\mathrm{N}_{2}$ $\mathrm{C}^{3} \Pi_{u}-\mathrm{B}^{3} \Pi_{g}$ electronic transition, located at about $380.5,357.6,337.1,316.4$ and $298.5 \mathrm{~nm}$ respectively. In addition, the spectral lines of $\mathrm{Cu}$ atoms referred to above were also ascertained.

In Fig. 6, it can be readily found that the emissions of different species appear in an orderly manner. As the discharges initiation, the emissions of activated $\mathrm{N}_{2}$ molecules first appeared in several 10-ns considered as the timescale of electron impact during the discharges pulse. And then, the emissions disappeared in a short period of time (about $300 \mathrm{~ns}$ in this experiment) with the rising of the $\mathrm{CN}$ violet band. Comparing with that of $\mathrm{N}_{2}$ molecules, the rising of $\mathrm{CN}$ violet band is rather slow. It took about $700 \mathrm{~ns}$ for the climbing while its declination also spent about $700 \mathrm{~ns}$. The timescale of the evolution is consistent with that of V-T energy transfer and chemical reactions in the dynamics of NPD. Besides, just like the $\mathrm{CN}$ violet band, the spectral lines of activated $\mathrm{H}$ and $\mathrm{O}$ atoms ( $\mathrm{H} \alpha$ at around $656 \mathrm{~nm}$ and $\mathrm{O}$ I triple lines at $777 \mathrm{~nm}$ ) also appear at about $100 \mathrm{~ns}$ after the discharges initiation with the declination of $\mathrm{N}_{2}$ molecules emissions. The effective lifetimes of the excited states of $\mathrm{H}$ and $\mathrm{O}$ atoms are 104 and 113 ns respectively as displayed in Fig. $6 \mathrm{~b}$.

Moreover, the transient evolution of the intermediate species shown by the time-resolved emission spectra in Fig. 6 also provided lots of critical informations for understanding and modifying the theoretical mechanisms of non-equilibrium plasma produced by NPD like the ultrafast heating mechanism of nanosecond pulsed discharges proposed by Popov [16]. As referred in the introduction, the mechanism declared that during the high voltage pulse, nitrogen molecules will first be excited by electron impact to electronic states such as $\mathrm{A}^{3} \Sigma, \mathrm{B}^{3} \Pi$, and $\mathrm{C}^{3} \Pi$. Then, the excited $\mathrm{N}_{2}$ molecules will be quenched by oxygen molecules producing atomic oxygen $\mathrm{O}\left({ }^{3} \mathrm{P},{ }^{1} \mathrm{~S}\right.$ and $\left.{ }^{1} \mathrm{D}\right)$ and ground-state $\mathrm{N}_{2}$ molecules within a few tens of nanoseconds. In the present experiment, the observed shortlife emissions which are originated from the $C^{3} \Pi_{u}-B^{3} \Pi_{g}$ electronic transition of $N_{2}$ molecules in initiation of the discharges indicated that nitrogen molecules were excited by electron impact to the upper electronic states $\mathrm{N}_{2}\left(\mathrm{C}^{3} \Pi_{u}\right)$, and then relaxed to $\mathrm{N}_{2}\left(\mathrm{~B}^{3} \Pi_{g}\right)$. Although the excited electronic states of nitrogen molecules were both mentioned in the ultrafast heating mechanism but the process $\mathrm{N}_{2}\left(\mathrm{C}^{3} \Pi_{u}\right) \rightarrow \mathrm{N}_{2}\left(\mathrm{~B}^{3} \Pi_{g}\right)$ was not referred. Then, the disappearance of $\mathrm{N}_{2}$ emission and the emergence of the $\mathrm{O}$ I triple spectral lines at the time around $100 \mathrm{~ns}$ after the discharges initiation also show some differences with the view of Popov because the O I triple spectral lines around $777 \mathrm{~nm}$ belong to $\left(3 p,{ }^{5} \mathrm{P}\right)$ $\left(3 p,{ }^{5} \mathrm{~S}\right)$ transition that did not appear in the ultrafast heating mechanism. However, according to the investigation of Germany [24], the $\left(3 p,{ }^{5} \mathrm{P}\right)$ excited states of O I could be produced via electron impact excitation of the $2 p^{4}\left({ }^{3} \mathrm{P}\right)$ ground state of atomic oxygen $\mathrm{O}$ which is one of products in the quenching step within tens of nanoseconds of Popov's mechanism. Therefore, it may be the way of producing the $\mathrm{O}\left(3 p,{ }^{5} \mathrm{P}\right)$ excited states. It should be noted that the simultaneous dissociative excitation of molecular oxygen could also produce $\mathrm{O}\left(3 p,{ }^{5} \mathrm{P}\right)$ at energies above the $\mathrm{e}+\mathrm{O}_{2} \rightarrow \mathrm{O}\left(3 p,{ }^{5} \mathrm{P}\right)$ threshold energy at $15.5 \mathrm{eV}$. But the cross section for this process is an order of magnitude smaller than the atomic excitation process [25]. And the time scale of the emergence of the O I triple spectral lines also indicates that the direct dissociative excitation of molecular oxygen by electron impact is less competitive. 
Furthermore, the spectral lines of excited $\mathrm{N}$ atoms and $\mathrm{N}$ ions observed in the emission spectra of NPD indicate that the nitrogen molecules were not only excited as declared in the ultrafast heating mechanism, but also were dissociated and ionized by the discharges. The violet bands of $\mathrm{CN}$ radicals further displayed that the $\mathrm{N}_{2}$ molecules, $\mathrm{N}$ atoms or $\mathrm{N}$ ions were involved in the reactions with $\mathrm{CH}_{4}$. Nevertheless, these indispensable intermediate species and the related reactions in NPD were not found in the existing kinetic simulations of the nanosecond pulsed discharges for methane/air combustion, such as GRI-Mech mechanism [26], 2-D kinetic simulations [27].

\section{The Determination of Temperature of CN Radicals}

As referred before, the appearance of $\mathrm{CN}$ violet emissions indicates the formation of gaseous $\mathrm{CN}$ radicals. It seems reasonable to assume that $\mathrm{CN}$ formation involves reactions between the $\mathrm{CH}_{4}$ fuel and the background active $\mathrm{N}_{2}$ molecules or their fragments (e.g. $\mathrm{C}_{2}$, $\mathrm{N}$ atoms etc.) produced by NPD in our case. As declared in some LIBS investigations of organic material $[28,29]$, the formation of $\mathrm{CN}$ occurs through the four-center reaction $\mathrm{C}_{2}+\mathrm{N}_{2} \rightarrow 2 \mathrm{CN}$ where nitrogen comes from ambient air. However, some other LIBS investigations [30,31] of hydrocarbons demonstrated that the often cited four-center reaction is very unlikely to be the major $\mathrm{CN}$ forming process in their LIBS experiments. Rather, the reaction of $\mathrm{C}$ and $\mathrm{N}_{2}$ appears to be responsible for the increasing $\mathrm{CN}$ concentration.

The investigation of $\mathrm{CN}$ violet emission has a great potential since $\mathrm{CN}$ can be detected in hydrocarbon fuel experiments in the presence of nitrogen. Furthermore, the signal-tonoise ratio of this molecular violet band is usually the highest in hydrocarbon fuel breakdown spectra. The information of the emission band and its relation to the molecular structure can disclose a deeper understanding of the principal formation routes of $\mathrm{CN}$ and other species existing in plasmas and useful information for quantitative measurement of combustion (e.g. temperature). As it is known that vibrational temperature provides the information of the energy transfer between electrons and heavy particles, especially molecules, which shows the similar tendency with the electron temperature. On the other hand, the rotational temperature is important to estimate gas temperature in atmospheric pressure plasmas due to the sufficiently fast rotational translational relaxation $[32,33]$.

Since the mean collisional frequency of $\mathrm{CN}$ in the atmosphere pressure is about $10^{9} / \mathrm{s}$ and the slow quenching of $\mathrm{CN} \mathrm{B}^{2} \Sigma^{+}$with methane, nitrogen et al. as referred in Ref. [34], although the $\mathrm{R}-\mathrm{T}$ relaxation of $\mathrm{CN}$ may need a few tens of collisions at high temperature, we can also expect that, in the present experiment, the rotational temperature of $\mathrm{CN}$ radicals has close relationship with the gas temperature in the plasma which has the most significant effect on chemical processes in fuel-air plasmas, in particular on the rates of chain branching fuel oxidation reactions. And it also affects the physical and chemical processes as well as the energy distribution and equilibrium condition exhibited in the plasma region. Therefore time-resolved and spatially resolved temperature measurements in these plasmas are extremely important for understanding the mechanism of plasmaassisted combustion [19]. Besides, the measurement of temperatures of $\mathrm{CN}$ radicals which is a signature of the chemical reaction between $\mathrm{CH}_{4}$ and $\mathrm{N}_{2}$ within the interaction of Bunsen flame and the NPD, especially of the rotational temperature, owns some significance for the heating load estimates related to discharges, understanding the formation mechanism of $\mathrm{CN}$ in plasma, and performing numerical simulation. In order to provide more experimental data for deeper understanding of the principal formation routes of $\mathrm{CN}$ and further numerical simulation for NPD, the measurement of time-resolved rotational 

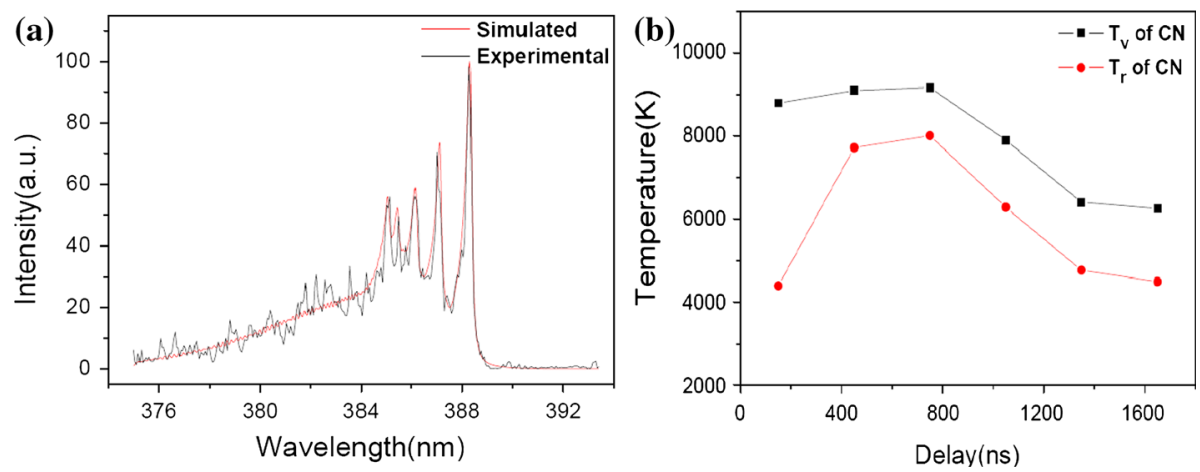

Fig. 7 a Experimental and simulated $C N$ emission spectra $\left(\mathrm{T}_{\mathrm{v}}=7900 \mathrm{k}, \mathrm{T}_{\mathrm{r}}=6280 \mathrm{k}\right.$, delay $=1050 \mathrm{~ns}$, gate $=300 \mathrm{~ns}$ ), $\mathbf{b}$ the time evolution of simulated vibrational and rotational temperatures of $\mathrm{CN}$ radicals (the delay time in figure was set as the delay of acquisition plus the half-width of ICCD gate)

and vibrational temperatures using $\mathrm{CN}$ radicals was performed here. Similar to Refs. [31, $35,36]$, the high temperature emission spectra of $\mathrm{CN}$ violet band in the plasma were recorded and analyzed. As shown in Fig. 7a, the rotational and vibrational temperatures of $\mathrm{CN}$ radicals were measured by simulating the experimental spectra with LIFBASE software, with considering the collisional broadening and Doppler broadening in Bunsen flame. The simulated temperatures and their time evolution with the delay time were displayed in Fig. 7b. For clarity, the delay time in the figure was set as the delay of the acquisition plus the half of ICCD exposure time.

Simulated temperatures of $\mathrm{CN}$ radicals exhibited in Fig. $7 \mathrm{~b}$ show that, in the rising process of $\mathrm{CN}$ violet band emission (delay: 0-700 ns, deduced from Fig. 6), the vibrational temperatures of $\mathrm{CN}$ radicals are rather high and fairly constant (around $9000 \mathrm{~K}$ ) while the rotational temperatures grow up from $4400 \mathrm{~K}$ to about $8000 \mathrm{~K}$. Similar temperatures were obtained from measurements of the CN spectra in laser-induced plasmas in Ref. [36], although in that case, no evidence of differences between rotational and vibrational temperatures was found. In this experiment, the growth of the rotational temperature manifests that the $\mathrm{CN}$ radicals may undergo an endothermic process with high initial vibrational energy in non-equilibrium plasma. During the declination of $\mathrm{CN}$ violet band emission (delay: 700-1500 ns), the vibrational and rotational temperatures are both decrease. It indicates that energy transference and relaxation of $\mathrm{CN}$ radicals happen in this period.

\section{Conclusions}

In conclusion, nanosecond pulsed discharges were applied to an experimental study for exploring the temporal response of a premixed methane/air Bunsen flame with two optical diagnostic methods. The microstructure and dynamic response of flame to the discharges was observed by a schlieren system, and the radial velocity of the shock wave was also measured. Time-resolved optical emission spectroscopy offered intermediate species, their evolutionary timescales and temperature in the plasma region. The experimental results provided lots of critical data for understanding the dynamics of non-equilibrium plasma produced by NPD and performing further numerical simulation. In addition, it also indicates that NPD can stir reactant gases and interact with flame by the generated shock wave 
and vortices while it produces more reactive plasma than LIBS. It makes the technique may be more suited for applications in combustion enhancement and instability control.

Acknowledgments The work is partially supported by National Science Foundation of China (Grant Nos. 11102215 and 91216101). The authors acknowledge the technical assistance provided by S. Z. Zhang at the Institute of Mechanics, Chinese Academy of Sciences.

\section{References}

1. Kim W, Do H, Mungal MG, Cappelli MA (2008) Optimal discharge placement in plasma-assisted combustion of a methane jet in cross flow. Combust Flame 15:3603

2. Starikovskiy A, Aleksandrov N (2013) Plasma-assisted ignition and combustion. Prog Energy Combust Sci 39:61

3. Starikovskaia SM (2014) Nanosecond discharges and development of kinetic mechanisms. J Phys D: Appl Phys 47:353001

4. Kim Y, Ferreri VW, Rosocha LA, Anderson GK (2006) Effect of plasma chemistry on activated propane/air flames. IEEE Trans Plasma Sci 34:2532

5. Wang F, Liu JB, Sinibaldi J, Brophy C, Kuthi A, Jiang C, Ronney P, Gundersen M (2005) Transient plasma ignition of quiescent and flowing air/fuel mixtures. IEEE Trans Plasma Sci 33:844

6. Babaritskii AI, Baranov IE, Bibikov MB, Demkin SA, Zhivotov VK, Konovalov GM, Lysov GV, Moskovskii AS, Rusanov VD, Smirnov RV, Chebankov FN (2004) Artial hydrocarbon oxidation processes induced by atmospheric pressure microwave discharge plasma. High Energy Chem 38:407

7. Yu Starikovskii A (2005) Plasma supported combustion. Proc Combust Inst 30:2405

8. Reinhard N (2012) Laser-induced breakdown spectroscopy fundamentals and applications. Springer, Heidelberg, Dordrecht, London, New York

9. Miziolek AW, Palleschi V, Schechter I (2006) Laser-induced breakdown spectroscopy: fundamentals and applications. Cambridge University Press, Cambridge

10. Ombrello T, Qin X, Ju Y, Gutsol A, Fridman A, Campbell C (2006) Combustion enhancement via stabilized piecewise nonequilibrium gliding arc plasma discharge. AIAA J 44:142

11. Sun W, Uddi M, Ombrello T, Won SH, Carter C, Ju Y (2011) Effects of non-equilibrium plasma discharge on counterflow diffusion flame extinction. Proc Combust Inst 33:3211

12. Starik AM, Kozlov VE, Titova NS (2010) On the influence of singlet oxygen molecules on the speed of flame propagation in methane-air mixture. Combust Flame 157:313

13. Ombrello T, Won SH, Ju Y, Williams S (2010) Flame propagation enhancement by plasma excitation of oxygen. Part II: Effects of $\mathrm{O}_{2}\left(\mathrm{a}^{1} \Delta_{\mathrm{g}}\right)$. Combust Flame 157:1916

14. Kosarev IN, Aleksandrov NL, Kindysheva SV, Starikovskaia SM, Yu Starikovskii A (2008) Kinetic mechanism of plasma-assisted ignition of hydrocarbons. J Phys D Appl Phys 41:032002

15. Aleksandrov NL, Kindysheva SV, Kosarev IN, Starikovskaia SM, Yu Starikovskii A (2009) Mechanism of ignition by non-equilibrium plasma. Proc Combust Inst 32:205

16. Popov NA (2001) Investigation of the mechanism for rapid heating of nitrogen and air in gas discharges. Plasma Phys Rep 27:886

17. Stancu GD, Kaddouri F, Lacoste DA, Laux CO (2010) Atmospheric pressure plasma diagnostics by OES, CRDS and TALIF. J Phys D Appl Phys 43:124002

18. Pai DZ, Lacoste DA, Laux CO (2010) Nanosecond repetitively pulsed discharges in air at atmospheric pressure-the spark regime. Plasma Sources Sci Technol 19:065015

19. Adamovich IV, Lempert WR (2015) Challenges in understanding and development of redictive models of plasma assisted combustion. Plasma Phys Control Fusion 57:014001

20. Settles GS (2001) Schlieren and shadowgraph techniques: visualizing phenomena in transparent media. Springer, New York

21. Aleksandrov NL, Kindysheva SV, Kukaev EN, Starikovskaya SM, Yu Starikovskii A (2009) Simulation of the ignition of a methane-air mixture by a high-voltage nanosecond discharge. Plasma Phys Rep 35:867

22. Xu DA, Lacoste DA, Rusterholtz DL, Elias P-Q, Stancu GD, Laux CO (2011) Experimental study of the hydrodynamic expansion following a nanosecond repetitively pulsed discharge in air. Appl Phys Lett 99:121502

23. Gottfried JL (2014) Influence of exothermic chemical reactions on laser-induced shock waves. Phys Chem Chem Phys 16(39):21452 
24. Germany GA, Anderson RJ, Salamo GJ (1988) Electron impact excitation of the $3 p\left({ }^{5} \mathrm{P}\right)$ state of atomic oxygen. J Chem Phys 89:1999

25. Kotzagianni M, Couris S (2013) Femtosecond laser induced breakdown spectroscopy of air-methane mixtures. Chem Phys Lett 561-562:36

26. Frenklach $\mathrm{M}$ et al. http://www.me.berkeley.edu/gri_mech/

27. Bak MS, Do H, Mungal MG, Cappelli MA (2012) Plasma-assisted stabilization of laminar premixed methane/air flames around the lean flammability limit. Combust Flame 159:3128

28. Vivien C, Hermann J, Perrone A, Boulmer-Leborgne C, Luches A (1998) A study of molecule formation during laser ablation of graphite in low-pressure nitrogen. J Phys D 31:1263

29. St-Onge L, Sing R, Béchard S, Sabsabi M (1999) Carbon emissions following $1.064 \mu \mathrm{m}$ laser ablation of graphite and organic samples in ambient air. Appl Phys A Mater Sci Process. 69:S913

30. Ma Q, Dagdigian PJ (2011) Kinetic model of atomic and molecular emissions in laser-induced breakdown spectroscopy of organic compounds. Anal Bioanal Chem 400:3193

31. Fernández-Bravo Á, Delgado T, Lucena P, Javier Laserna J (2013) Vibrational emission analysis of the $\mathrm{CN}$ molecules in laser-induced breakdown spectroscopy of organic compounds. Spectrochim Acta Part B 89:77

32. Moon SY, Kim DB, Gweon B, Choe W (2008) Spectroscopic characterization of rovibrational temperatures in atmospheric pressure $\mathrm{He} / \mathrm{CH}_{4}$ plasmas. Phys Plasmas 15:103504

33. Pellerin S, Cormier JM, Richard F, Musiol K, Chapelle J (1996) A spectroscopic diagnostic method using UV OH band spectrum. J Phys D 29:726

34. Jackson WM, Faris JL (1972) Quenching of the $\mathrm{B}^{2} \Sigma^{+}$state of the CN radical. J Chem Phys 56:95

35. Parigger CG, Woods AC, Surmick DM, Gautama G, Witte MJ, Hornkohl JO (2015) Computation of diatomic molecular spectra for selected transitions of aluminum monoxide, cyanide, diatomic carbon, and titanium monoxide. Spectrochim Acta Part B 107:132

36. Hornkohl JO, Parigger C, Lewis JWL (1991) Temperature measurements from CN spectra in a laserinduced plasma. J Quant Spectrosc Radiat Transf 46:405 